BIRKENMEIER (Jochen), VELTMANN (Claus) (dir.), Kinder, Krätze, Karitas. Waisenhäuser in der frühen Neuzeit

Halle : Verlag der Franckeschen Stiftungen zu Halle, 2009, 232 p.

(Kataloge der Franckeschen Stiftungen, 23)

Jean-Luc Le Cam

\title{
OpenEdition
}

Journals

Édition électronique

URL : https://journals.openedition.org/histoire-education/2166

DOI : 10.4000/histoire-education.2166

ISSN : 2102-5452

Éditeur

ENS Éditions

Édition imprimée

Date de publication : 1 juillet 2010

Pagination : $95-98$

ISSN : 0221-6280

Référence électronique

Jean-Luc Le Cam, «BIRKENMEIER (Jochen), VELTMANN (Claus) (dir.), Kinder, Krätze, Karitas.

Waisenhäuser in der frühen Neuzeit », Histoire de l'éducation [En ligne], 127 | 2010, mis en ligne le 10

mars 2011, consulté le 20 mai 2021. URL : http://journals.openedition.org/histoire-education/2166 ;

DOI : https://doi.org/10.4000/histoire-education.2166

Ce document a été généré automatiquement le 20 mai 2021.

(c) Tous droits réservés 


\section{BIRKENMEIER (Jochen), VELTMANN (Claus) (dir.), Kinder, Krätze, Karitas. Waisenhäuser in der frühen Neuzeit}

Halle : Verlag der Franckeschen Stiftungen zu Halle, 2009, 232 p.

(Kataloge der Franckeschen Stiftungen, 23)

Jean-Luc Le Cam

\section{RÉFÉRENCE}

BIRKENMEIER (Jochen), VELTMANN (Claus) (dir.), Kinder, Krätze, Karitas. Waisenhäuser in der frühen Neuzeit, Halle : Verlag der Franckeschen Stiftungen zu Halle, 2009, 232 p.

(Kataloge der Franckeschen Stiftungen, 23)

1 L'allitération du titre de ce catalogue d'exposition est un clin d'œil aux fameux « trois $\mathrm{K} »$ (Kinder, Küche, Kirche : enfants, cuisine, église), par lesquels le discours conservateur, voire réactionnaire, voulait définir la place dévolue aux femmes en Allemagne. L'analogie, cependant, tourne court, puisque le titre dénote au contraire une situation de tension entre réalité et intention, à savoir celle des enfants des orphelinats pris entre la gale, synonyme de leur condition précaire et de leur mal-vivre, et la charité, censée être le but et le fondement de l'institution qui leur permettait de survivre.

2 On ne saurait trouver de lieu plus emblématique de cette problématique que la fondation Francke ${ }^{1}$ à Halle, héritière de la création en 1698, par le piétiste August Hermann Francke, d'un grand orphelinat qui se développa en trois décennies jusqu'à devenir une véritable cité scolaire attirant, au-delà des orphelins, des élèves de toute l'Europe. Depuis la chute du mur, l'Allemagne s'efforce avec un certain succès de faire revivre ce patrimoine extraordinaire, malheureusement délaissé dans la seconde moitié du XXe siècle, grâce à diverses subventions et en restituant à ces fondations l'autonomie qu'elles avaient perdue dans la RDA. L'ensemble de quelque 50 bâtiments, sur 14 hectares, a été classé au patrimoine de l'humanité par l'Unesco et 
progressivement restauré pour héberger différentes institutions respectant la tradition du lieu : écoles, université, centre biblique, institutions sociales et culturelles. Mais reste bien sûr, au centre de ce dispositif, le premier orphelinat historique, conservé comme musée et lieu d'exposition, ainsi que la splendide bibliothèque, restituée à son état de $1746^{2}$.

C'est en 1948 que l'institut accueillit les derniers enfants avant de fermer ses portes. Lors de la renaissance des années 1990, on se posa la question du maintien d'une tradition d'encadrement social de la jeunesse. Celle-ci ne pouvait évidemment plus prendre les mêmes formes que celles de l'ancien établissement ${ }^{3}$. On lui substitua des centres d'accueil pour enfants et adolescents en difficulté, ou de compétences familiales pour l'éducation et la santé. Pour autant, les fondations Francke entendent affronter les problèmes sociaux contemporains en gardant la conscience de leurs racines historiques. C'est pourquoi elles encouragent la recherche et la diffusion de connaissances sur ce que furent ces institutions à l'origine. C'est le sens de cette très belle exposition, structurée en sept chapitres mettant à profit autant d'espaces et présentant successivement : l'historique de la prise en charge des orphelins jusqu'au XVIe siècle; l'histoire des orphelinats aux XVIIe et XVIIIe siècles; les orphelinats de l'âge d'or hollandais, modèles des orphelinats allemands; la fondation faite par August Hermann Francke à Halle; l'extraordinaire rayonnement de l'orphelinat de Halle en Allemagne et à l'étranger, jusque dans les colonies ; les critiques des Lumières connues sous le nom de "querelle des orphelinats"; enfin, la prise en charge contemporaine de ces enfants dans des structures d'un nouveau type aux XIXe et XXe siècles («orphelins d'hier et d'aujourd'hui »).

L'ouvrage se présente certes, dans ses cent dernières pages, comme un catalogue traditionnel d'exposition, avec une notice pour chaque objet présenté, dont les plus remarquables sont reproduits. Mais l'intérêt du livre est plus encore dans les cent trente pages qui précèdent et qui constituent un véritable recueil collectif sur la question des orphelinats à l'époque moderne, également très bien illustré. Ces textes reprennent le plan de l'exposition avec quelques variantes et plus de richesse. Claus Veltmann, conservateur du musée de l'orphelinat, retrace l'histoire de la prise en charge des orphelins depuis l'Antiquité tardive jusqu'au XVIe siècle. Pour la période moderne, il exploite notamment des travaux anciens sur Nuremberg ou la thèse de l'Américain Thomas Max Safley sur Augsbourg. La Réforme n'a guère apporté de changements dans ce domaine, du moins en milieu urbain. En revanche, la suppression des monastères ruraux a obligé à une réorganisation de la prise en charge des orphelins. Manfred Eder présente le cas particulier des orphelinats catholiques à l'époque moderne sous le titre-citation emblématique « On doit leur apprendre à lever leurs petits yeux et leurs petites mains vers le ciel ». À travers différents exemples, pris en majorité au XVIIIe siècle, il en arrive à la conclusion qu'il n'y avait pas de différence fondamentale sur cette question en fonction des confessions. Certes, l'éducation religieuse se faisait dans le dogme propre à chacune, ici avec une assez forte coloration jésuitique, mais la philosophie globale et les systèmes d'organisation étaient très semblables. Karl Härter s'attache à présenter la prise en charge des orphelins et la gestion des orphelinats dans le contexte de la " police » moderne (Policey), au sens que ce mot avait alors. C'est la problématique de la discipline sociale qui est ici abordée dans le cadre de la construction de l'État moderne, ce qui correspond, au particularisme allemand de la principauté territoriale près, au débat français des années 1970-1980 sur le "grand enfermement». Jokes Spaans livre un bref article en anglais sur les 
orphelinats hollandais, qui correspond tout à fait à la troisième salle de l'exposition. Nombreuses et bien financées grâce à la prospérité hollandaise, bien organisées grâce au sens de l'ordre et à la discipline calviniste, ces institutions ont pu passer au XVIIe siècle pour des modèles en Europe.

5 Udo Sträter a la tâche ingrate, sinon impossible, de faire en une dizaine de pages le résumé de l'histoire de la fondation hébergeant l'exposition, qui a évidemment suscité depuis longtemps toutes sortes de travaux et notamment, dans la dernière décennie, un programme intensif de recherche financé par la Deutsche Forschungsgemeinschaft (DFG) ${ }^{4}$ pour saisir et exploiter les très nombreuses données sur les élèves et le personnel de ces écoles ${ }^{5}$. Il est vrai que l'orphelinat lui-même ne constitue qu'un élément des nombreuses formations scolaires qui se sont développées à Halle. On a d'ailleurs reproché à Francke, en son temps, de se servir du paravent de cette institution caritative pour développer, en fait, essentiellement un centre de diffusion du piétisme et un établissement scolaire privé. Ce thème est donc traité aussi dans les deux articles suivants, qui décrivent le rayonnement de l'orphelinat de Halle dans tout l'Empire (Antje Fasshauer) et à travers le monde (Jochen Birkenmeier). Ces deux contributions permettent de bien saisir le caractère tout à fait exceptionnel de cette œuvre et son succès international. La diffusion s'est faite essentiellement par imitation du modèle de Halle et par transfert de personnel enseignant formé dans les établissements entourant l'orphelinat. En revanche, les structures institutionnelles dépendaient à chaque fois des créateurs locaux des établissements, publics ou privés. L'époque des Lumières (Christina Vanja) vit s'accumuler les critiques contre la plupart des structures d'enfermement et d'éducation, au nom du droit au bonheur de l'individu et de l'idéal d'harmonie de la société. Outre la discipline et le manque de liberté, les conditions matérielles et d'hygiène de ces établissements sont particulièrement mises en cause : c'est là qu'on voit surgir le problème de la gale, particulièrement répandue chez les orphelins en raison notamment de leur emploi traditionnel au travail de la laine, mais aussi celui du rachitisme, du scorbut, sans parler des maladies infectieuses qui provoquaient une forte mortalité. Le plus éloquent et connu de ces critiques fut Johann Peter Süssmilch. Cette "querelle des orphelinats » provoqua une série de fermetures ou de rénovations d'établissements dans tout l'Empire, synthétisées sur une carte (p. 122). La dernière contribution, de Norbert Friedrich, clôt cette partie par une évocation du destin des orphelinats à l'époque contemporaine. Malgré une préférence croissante pour le placement en famille, ils restèrent des lieux d'accueil dont on ne put se passer, en particulier en 1945, lorsqu'il fallut prendre en charge les nombreuses populations de réfugiés et de refoulés venant de l'Est.

6 Au total, un ouvrage captivant, au contenu bien plus riche qu'un traditionnel catalogue d'exposition, pour autant magnifiquement illustré, et qui fera sans doute regretter à ses lecteurs lointains de ne pas avoir pu visiter cette exposition qui a fermé ses portes le 4 octobre 2009. 


\section{NOTES}

1. On parle plus exactement, en allemand, des fondations Francke pour désigner les différents établissements qu'il a fondés, ainsi que les dispositifs qui les financent.

2. Cf. http://www.francke-halle.de/main/index.php

3. La formule des villages d'enfants, illustrée p. 223, ou des placements en famille d'accueil est maintenant préférée.

4. Organisme allemand de financement de la recherche.

5. Menés sous la direction de Juliane Jacobi et Thomas J. Müller-Bahlke, ces travaux forment aussi le point de départ des thèses d'Axel Oberschelp et Silke Brockerhoff. 